\title{
GENERALIZED BETA-EXPONENTIAL WEIBULL DISTRIBUTION AND ITS APPLICATIONS
}

\section{N. I. BADMUS ${ }^{1}$, OLANREWAJU FAWEYA ${ }^{2}$}

and K. A. ADELEKE ${ }^{3}$

${ }^{1}$ Department of Mathematics

University of Lagos

Akoka

Nigeria

e-mail: nibadmus@unilag.edu.ng

2Department of Statistics

Ekiti State University

Ado-Ekiti, Ekiti State

Nigeria

${ }^{3}$ Department of Mathematics

Obafemi Awolowo University

Ile-Ife, Osun State

Nigeria

2020 Mathematics Subject Classification: 62E20, 62F07, 62G30, 62P10.

Keywords and phrases: moments, information matrix, order statistics, quantile function, Renyi entropy, $T-X$ distribution.

Received October 10, 2020; Revised November 11, 2020

(C) 2020 Scientific Advances Publishers

This work is licensed under the Creative Commons Attribution International License (CC BY 3.0).

http://creativecommons.org/licenses/by/3.0/deed.en_US

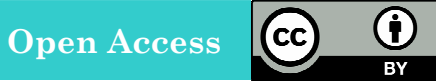




\begin{abstract}
In this article, we investigate a distribution called the generalized betaexponential Weibull distribution. Beta exponential $x$ family of link function which is generated from family of generalized $T-X$ distributions is used in generating the new distribution. Its density and hazard functions have different shapes and contains special case of distributions that have been proposed in literature such as beta-Weibull, beta exponential, exponentiatedWeibull and exponentiated-exponential distribution. Various properties of the distribution were obtained namely; moments, generating function, Renyi entropy and quantile function. Estimation of model parameters through maximum likelihood estimation method and observed information matrix are derived. Thereafter, the proposed distribution is illustrated with applications to two different real data sets. Lastly, the distribution clearly shown that is better fitted to the two data sets than other distributions.
\end{abstract}

\title{
1. Introduction
}

Generalized beta distributions have been widely developed by several authors and researchers in literature which is a way of introducing shape parameters to govern both skewness and vary tail weight. These distributions are more flexible, robust and fitting for specific real life data sets. In generalized distributions/models, the baseline distribution can be obtained from the proposed distribution/model when setting one or more parameters of the distribution to one (1). Morais et al. [20] extended generalized logistics to beta generalized logistic distribution by introducing two extra shape parameters to provide greater flexibility in modelling observed data. Zea et al. [33] studied and introduced beta exponentiated Pareto distribution with the hope that it will be useful and applied in economics. Several beta generalized type distributions were developed, see, for example, Cordeiro et al. [9], Singla et al. [32], Mahmoudi [19], Cordeiro and Castro [8], Barreto-Souza et al. [5] and Pescim et al. [29].

Meanwhile, exponential and Weibull distributions are continuous probability distributions widely and most commonly used for analyzing and modelling life time phenomenon and waiting time events. Research has been carried out by researchers on various types of exponential and Weibull distributions such as Weibull exponential distribution by 
Oguntunde et al. [25], exponentiated Weibull distribution by Kim et al. [16], Ashour and Afify [4], Elshathat [10] and Nassar and Eissa [23, 24], generalized exponential distribution include Okoli et al. [26], Olapade [27], Gupta and Kundu [12] to mention a few. Then, in the case of exponentiated $T-X$ distribution, Alzaghal et al. [3] and Alzaatrech et al. [2] but Alzaatrech proposed a new method for generating many new distributions using $T-X$ family of distribution. Therefore, motivated by the works of Hamdy and Mahmoud [13] extended a Weibull-exponential distribution which was generated from family of generalized $T-X$ distribution called generalized Weibull-exponential distribution.

The main aim of this article is to introduce a distribution called the generalized beta-exponential Weibull distribution with beta exponential $x$ family of link function which is generated from family of generalized $T-X$ distributions used in generating the new distribution In the proposed distribution, some special cases were generated when one or more parameters is equate to either 1 or 2 . Some of its properties also obtained include; survival, hazard rate, reverse hazard rate function, moments, generating function, Renyi entropy and quantile function.

Here, let $X$ be a random variable taken from Weibull distribution with probability density function (pdf) and cumulative distribution function (cdf), respectively, $f(x)=\gamma \theta x^{\theta-1} e^{-\gamma x^{\theta}} x>0$ and $F(x)=1-e^{-\gamma x^{\theta}}$. Then, let $T$ be a random variable taken from beta exponential distribution with pdf $f(t)=\frac{\lambda e^{-\lambda \beta x}\left(1-e^{-\lambda x}\right)^{\alpha-1}}{B(\alpha, \beta)}$. Therefore, the pdf of the beta exponential-Weibull distribution is

$$
\begin{aligned}
f(x) & =\frac{\lambda f(x)}{B(\alpha, \beta)}(1-F(x))^{\lambda \beta-1}\left(1-(1-F(x))^{\lambda}\right)^{\alpha-1} \\
& =\frac{\lambda \gamma \theta x^{\theta-1} e^{-\gamma x^{\theta}}}{B(\alpha, \beta)}\left(1-\left(1-e^{-\gamma x^{\theta}}\right)\right)^{\lambda \beta-1}\left(1-\left(1-\left(1-e^{-\gamma x^{\theta}}\right)\right)^{\lambda}\right)^{\alpha-1},
\end{aligned}
$$

$x>0, \alpha, \beta, \lambda, \gamma \& \theta>0$. See Alzaatreh et al. [2] and Hamdy and Mahmoud [13]. 
The rest of the paper is divided as follows: The generalized beta exponential-Weibull (GBEW) distribution is defined in Section 2. Various properties such as moments, generating function, quantile function, Renyi entropy, skewness, kurtosis and order statistics are discussed in Section 3. In Section 4, estimation of model parameters and observed information matrix are derived. The new distribution is illustrated with an application to real life data set and comparison of the result for the GBEW distribution and other known distribution is done. Section 5 contains the conclusion.

\section{Material and Method}

\subsection{The generalized beta exponential-Weibull (GBEW) distribution}

Alzaghal et al. [3] investigated and presented the pdf of exponentiated $T-X$ distribution as follows:

$$
\begin{aligned}
& f(x)=\frac{c \lambda}{B(\alpha, \beta)} f(x) F^{c-1}(x)\left(1-F^{c}(x)\right)^{\lambda \beta-1}\left(1-\left(1-F^{c}(x)\right)^{\lambda}\right)^{\alpha-1}, \\
& f(x)=\frac{c \lambda \gamma \theta x^{\theta-1} e^{-\gamma x^{\theta}}\left(1-e^{-\gamma x^{\theta}}\right)^{c-1}}{B(\alpha, \beta)}\left(1-\left(1-e^{-\gamma x^{\theta}}\right)^{c}\right)^{\lambda \beta-1} \\
& \times\left(1-\left(1-\left(1-e^{-\gamma x^{\theta}}\right)^{c}\right)^{\lambda}\right)^{\alpha-1},
\end{aligned}
$$

where $(\alpha, \beta, c$ and $\gamma)$ and $(\lambda, \theta)$ are shape and scale parameters, and Equation (4) becomes the density of the GBEW distribution.

From the proposed distribution (GBEWD), some special cases emanated when one or more parameters is equated to 1 or 2 and some of them are known distributions in literature as shown and stated in Table 1 below: 
Table 1. Summary of the Special cases of GBEW distribution

\begin{tabular}{lccccccl}
\hline Special Case & $\alpha$ & $\beta$ & $c$ & $\gamma$ & $\lambda$ & $\theta$ & Author \\
\hline BW Distribution & -- & -- & 1 & -- & 1 & -- & Famoye et al. [11] and Lee et al. [17] \\
BE Distribution & -- & -- & 1 & -- & 1 & 1 & Nadarajah and Kotz [22] \\
EE Distribution & 1 & 1 & -- & -- & -- & 1 & Gupta and Kundu [12] \\
EW Distribution & -- & 1 & -- & -- & 1 & -- & Abo-Kasem [30] and Choudhury [7] \\
ER Distribution & 1 & 1 & 1 & -- & 1 & 2 & Mahmoud and Ghazal [18] \\
EER Distribution & 1 & 1 & -- & -- & -- & 2 & \\
BR Distribution & -- & -- & 1 & -- & 1 & 2 & \\
\hline
\end{tabular}

However, the corresponding cdf of GBEW distribution is obtained as follows:

$$
\begin{aligned}
F(x)=P(X \leq x) & =\int_{0}^{x} f(x) d x=\int_{0}^{x} \frac{c \lambda \gamma \theta x^{\theta-1} e^{-\gamma x^{\theta}}\left(1-e^{-\gamma x^{\theta}}\right)^{c-1}}{B(\alpha, \beta)} \\
& \times\left(1-\left(1-e^{-\gamma x^{\theta}}\right)^{c}\right)^{\lambda \beta-1}\left(1-\left(1-\left(1-e^{-\gamma x^{\theta}}\right)^{c}\right)^{\lambda}\right)^{\alpha-1} .
\end{aligned}
$$

Let

$$
\begin{gathered}
U=\left(1-\left(1-e^{-\gamma x^{\theta}}\right)^{c}\right)^{\lambda}, d u=c \lambda \gamma \theta x^{\theta-1} e^{-\gamma x^{\theta}}\left(1-e^{-\gamma x^{\theta}}\right)^{c-1} d x, \\
F(x)=\frac{1}{B(\alpha, \beta)} \int_{0}^{F(u)} U^{\beta-1}(1-U)^{\alpha-1} d u, \\
F(x)=\frac{B_{F(u)}(\alpha, \beta)}{B(\alpha, \beta)}=I_{\left[1-\left(1-e^{-\gamma x}\right)^{c}\right]^{\lambda}}(\alpha, \beta) .
\end{gathered}
$$


Also, survival function (Sur), hazard rate function (hz), reverse rate function (rhz), and cumulative hazard rate function (chz) are given below respectively.

$$
\begin{aligned}
& \operatorname{Sur}_{(u ; \alpha, \beta)}=1-I_{F(u)}(\alpha, \beta)=\frac{B(\alpha, \beta)-I_{\left.\left[1-\left(1-e^{-\gamma x}\right)^{c}\right]^{\lambda}\right]^{\lambda}(\alpha, \beta)}}{B(\alpha, \beta)}, \\
& h z_{(u ; \alpha, \beta)}=\frac{f(u ; \alpha, \beta)}{1-I_{F(u)}(\alpha, \beta)}=\frac{f(u) F(u)^{\beta-1}[1-F(u)]^{\alpha-1}}{B(\alpha, \beta)-I_{\left[1-\left(1-e^{-\gamma x}\right)^{c}\right]^{\lambda}}(\alpha, \beta)} \\
& r h z_{(u ; \alpha, \beta)}=\frac{f(u ; \alpha, \beta)}{I_{F(u)}(\alpha, \beta)}=\frac{f(u)[F(u)]^{\beta-1}[1-F(u)]^{\alpha-1}}{\left.\left.I_{\left[1-\left(1-e^{-\gamma x} \theta\right.\right.}\right)^{c}\right]^{\lambda(\alpha, \beta)}},
\end{aligned}
$$

and

$$
\begin{aligned}
\operatorname{ch} z_{(u ; \alpha, \beta)} & =-\log [\operatorname{Sur}(u ; \alpha, \beta)] \\
& =-\log \left[\frac{B(\alpha, \beta)-I_{\left[1-\left(1-e^{-\gamma x}\right)^{c}\right]^{\lambda}}\left[\frac{(\alpha, \beta)}{B(\alpha, \beta)}\right] .}{} .\right.
\end{aligned}
$$

See Handique and Chakraborty [14]. 
The shapes of the pdf and hazard function of GBEW distribution are plotted below:

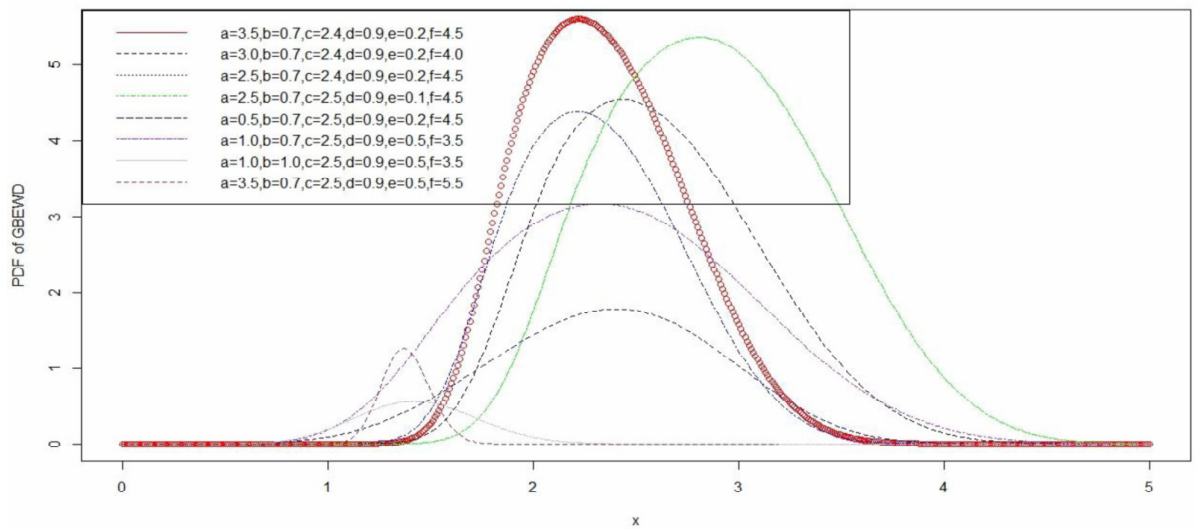

(i)

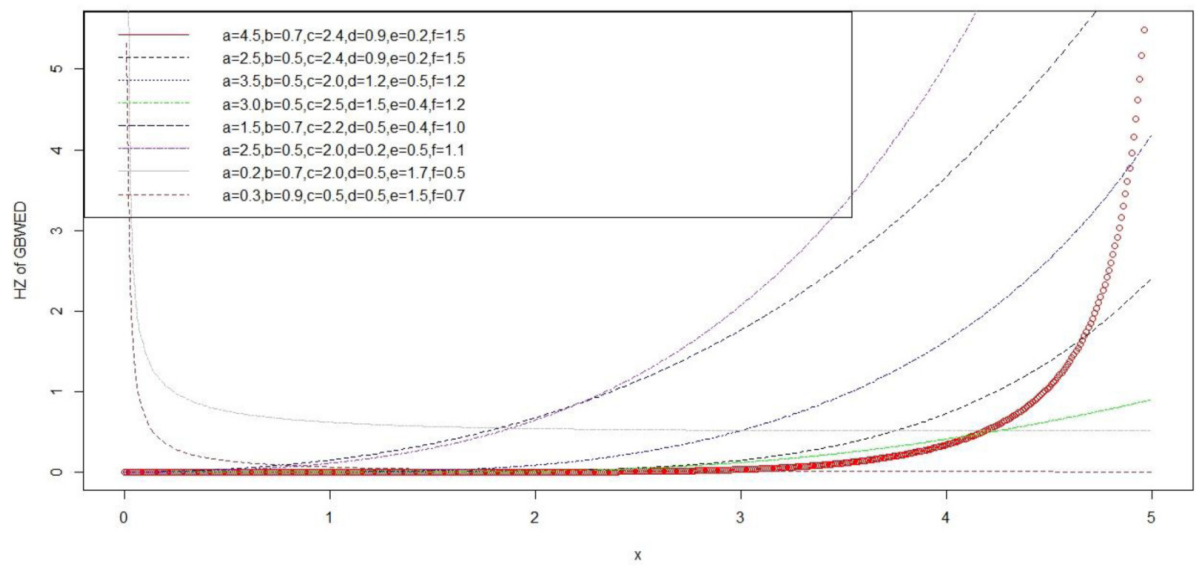

(ii)

Figure 1. Plots of the GBEW densities in (i), where $(a, b, c, d, e, f)$ $=(\alpha, \beta, c, \lambda, \gamma, \theta)$ for various values, i.e., $(3.5,0.7,2.4,0.9,0.2,4.5)$, $(3.0,0.7,2.4,0.9,0.2,4.0)$ and hazard function in (ii) $(a, b, c, d, e, f)=$ $(\alpha, \beta, c, \lambda, \gamma, \theta)$ for different values, i.e., (4.5, $0.7,2.4,0.9,0.2,1.5)$, $(2.5,0.5,2.4,0.9,0.2,1.5)$ etc. 


\section{Moment Generating Function}

The moment generating function (mgf) of GBEW distribution is expressed as follows:

$$
M_{x}(s)=E\left(e^{s x}\right)=\int_{-\infty}^{\infty} e^{s x} f(x ; \alpha, \beta, c, \lambda, \gamma, \theta) d x
$$

This can be written in terms of exponentiated-Weibull distribution

$$
=\frac{1}{B(\alpha, \beta)} \int_{-\infty}^{\infty} e^{s x} \frac{(-1)^{j+1}}{(j+\beta)}\left(\begin{array}{c}
\alpha-1 \\
j
\end{array}\right) \frac{d}{d x}[\operatorname{Sur}(x ; c, \lambda, \gamma, \theta)]^{j+\beta} d x
$$

By calculating the integral, we have

$$
=\frac{1}{B(\alpha, \beta)(j+\beta)} \sum_{j=0}^{\alpha-1}(-1)^{j+1}\left(\begin{array}{c}
\alpha-1 \\
j
\end{array}\right) \int_{-\infty}^{\infty} e^{s x} \frac{d}{d x}[\operatorname{Sur}(x ; c, \lambda, \gamma, \theta)]^{j+\beta} d x,
$$

where $\int_{-\infty}^{\infty} e^{s x} \frac{d}{d x}[\operatorname{Sur}(x ; c, \lambda, \gamma, \theta)]^{j+\beta} d x$ is the moment generating function (mgf) of the exponentiated Weibull distribution Pal et al. [28] and is given as:

$$
E\left(Z^{s}\right)=c \lambda^{-s} \Gamma\left(\frac{s}{\gamma}+1\right) \sum_{i=0}^{c-1}\left(\begin{array}{c}
c-1 \\
i
\end{array}\right)(-1)^{i}(i+1)^{\frac{S}{\gamma}+1} .
$$

This implies that (11) can also be written as:

$$
\begin{aligned}
M_{x}(s) & =\frac{1}{B(\alpha, \beta)(j+\beta)} \sum_{j=0}^{\alpha-1}(-1)^{j+1}\left(\begin{array}{c}
\alpha-1 \\
j
\end{array}\right) c \lambda^{-s} \Gamma\left(\frac{s}{\gamma}+1\right) \sum_{i=0}^{c-1}\left(\begin{array}{c}
c-1 \\
i
\end{array}\right)(-1)^{i}(i+1)^{\frac{S}{\gamma}+1} \\
& =\frac{c \lambda^{-s}}{B(\alpha, \beta)(j+\beta)} \Gamma\left(\frac{s}{\gamma}+1\right) \sum_{i=0}^{c-1} \sum_{j=0}^{\alpha-1}(-1)^{(i+j)+1}\left(\begin{array}{c}
c-1 \\
i
\end{array}\right)\left(\begin{array}{c}
\alpha-1 \\
j
\end{array}\right)(i+1)^{\frac{S}{\gamma}+1}
\end{aligned}
$$


The $s$-th moment of GBEW distribution is written from (12) as:

$\mu_{s}^{\prime}=E\left(X^{s}\right)=\frac{c \lambda^{-s}}{B(\alpha, \beta)(j+\beta)} \Gamma\left(\frac{s}{\gamma}+1\right) \sum_{i=0}^{c-1} \sum_{j=0}^{\alpha-1}(-1)^{(i+j)+1}\left(\begin{array}{c}c-1 \\ i\end{array}\right)\left(\begin{array}{c}\alpha-1 \\ j\end{array}\right)(i+1)^{\frac{S}{\gamma}+1}$.

Setting $\alpha=\beta=1$ in (13) gives the $s$-th moment of the baseline distribution by Pal et al. [28].

$$
\mu_{s}^{\prime}=E\left(X^{s}\right)=c \lambda^{-s} \Gamma\left(\frac{s}{\gamma}+1\right) \sum_{i=0}^{c-1}\left(\begin{array}{c}
c-1 \\
i
\end{array}\right)(-1)^{i}(i+1)^{\frac{S}{\gamma}+1} .
$$

\subsection{Renyi entropy}

The Renyi entropy is defined as:

$$
I_{R}=(\delta)=\frac{1}{(1-\delta)} \log \left(\int_{-\infty}^{\infty} f(x)^{\delta} d x\right)
$$

where $\delta>0$ and $\delta \neq 1$ by using binomial expression in (4) as it was used in Handique and Chakraborty [14] expressed as:

$$
\begin{aligned}
f(x) & =(x ; \alpha, \beta, c, \lambda, \gamma, \theta)^{\delta} \\
& =\left[[B(\alpha, \beta)]^{-1} c \lambda \gamma \theta f(x) F(x)^{c-1}\left[1-F(x)^{c}\right]^{\lambda \beta-1}\right]^{\delta}\left[1-\left(1-F(x)^{c}\right)^{\lambda}\right]^{(\alpha-1)^{\delta}} \\
& =\left[\frac{c \lambda \gamma \theta x^{\theta-1} e^{-\gamma x^{\theta}}\left(1-e^{-\gamma x^{\theta}}\right)^{c-1}}{B(\alpha, \beta)}\left[1-\left(1-e^{-\gamma x^{\theta}}\right)^{c}\right]^{\lambda \beta-1}\right]^{\delta} \\
& \times\left[1-\left(1-\left(1-e^{-\gamma x^{\theta}}\right)^{c}\right)^{\lambda}\right]^{(\alpha-1)^{\delta}}
\end{aligned}
$$




$$
\begin{aligned}
=\frac{1}{B(\alpha, \beta)^{\delta}}\left[c \lambda \gamma \theta f(x) F(x)^{c-1}\left[1-F(x)^{c}\right]^{\lambda \beta-1}\right]^{\delta} & \\
& \times \sum_{a=0}^{\alpha-1}\left(\begin{array}{c}
(\alpha-1)^{\delta} \\
a
\end{array}\right)(-1)^{a}\left[1-F(x)^{c}\right]^{\lambda a} .
\end{aligned}
$$

Therefore, the Renyi entropy of $X$ is obtained as

$$
\begin{aligned}
I_{R}(\delta)= & \frac{1}{(1-\delta)} \log \left[\sum_{a=0}^{(\alpha-1) \delta} R_{a} \int_{0}^{\infty}\left[c \lambda f(x) F(x)^{c-1}\left[1-F(x)^{c}\right]^{\lambda \beta-1}\right]^{\delta}\left[1-F(x)^{c}\right]^{\beta a} d x\right] \\
I_{R}(\delta)= & \frac{1}{(1-\delta)} \\
& \log \left[\sum_{a=0}^{(\alpha-1) \delta} R_{a} \int_{0}^{\infty}\left[c \lambda \gamma \theta x^{\theta-1} e^{-\gamma x^{\theta}}\left(1-e^{-\gamma x^{\theta}}\right)^{c-1}\left[1-\left(1-e^{-\gamma x^{\theta}}\right)^{c}\right]^{\lambda \beta-1}\right]^{\delta}\right. \\
\times & \left.\times\left[1-\left(1-\left(1-e^{-\gamma x^{\theta}}\right)^{c}\right)^{\lambda}\right]^{\beta a} d x\right] \\
= & \frac{1}{1-\delta} \log \left[\sum_{a=0}^{(\alpha-1) \delta} R_{a} \int_{0}^{\infty}[f(x ; c, \lambda, \beta)]^{\delta}[\operatorname{Sur}(x ; c, \lambda)]^{a} d x\right]
\end{aligned}
$$

where $R_{a}=\frac{1}{B(\alpha, \beta)^{\delta}}\left(\begin{array}{c}(\alpha-1)^{\delta} \\ a\end{array}\right)(-1)^{a}, f(x ; c, \lambda, \gamma, \theta)$ and $\operatorname{Sur}(x ; c, \lambda, \gamma, \theta)$ are the pdf and survival function of the exponentiated-Weibull distribution, and (14) becomes the Renyi entropy of the GBEW distribution. 


\subsection{Quantile function}

The quantile function of $X$ associating to (8) is given as

$$
Q(u)=F^{-1}(u)=\frac{\beta^{-1}}{\left(I_{u}^{-1}(\alpha, \beta)^{\frac{1}{c \lambda}}\right)^{\frac{1}{\theta}}}, \quad 0<u<1,
$$

where $I_{u}^{-1}(\alpha, \beta)$ is the inverse of the incomplete beta function and the function $I_{u}^{-1}(\alpha, \beta)$ is expressed as a power series

$$
I_{u}^{-1}(\alpha, \beta)=\sum_{j=1}^{\infty} q i[\alpha, B(\alpha, \beta) u]^{\frac{1}{c \lambda}}
$$

where $q i=1$ and the remaining coefficients satisfy the following recursion:

$$
\begin{aligned}
& q i=\frac{1}{i^{2}+(\alpha-2) i+(1-\alpha)}\left\{\left(1-\varphi_{i}, 2 \sum_{r=2}^{i-1} q m q i+1-n[m(1-\alpha)(i-m)-m\right.\right. \\
& \left.(m-1)]+\sum_{m=1}^{i-1} \sum_{n=1}^{i-m} q m q n q i+1-m-n[m(m-\alpha)+n(\alpha+\beta-2)(i+1-m-n)]\right\},
\end{aligned}
$$

see Zea et al. [33]. Where $\varphi_{i}, 2=1$ if $i=2$ and $\varphi_{i}, 2=0$ if $i \neq 2$.

Following Zea et al. [33], the importance and motivation to use quantile-based measures are robust and less sensitive to outliers and exist even for distributions without moments. The skewness and kurtosis of the GBEW distribution based on the Bowley's skewness which based on quantiles Kenney and Keeping [15]:

$$
S K(B)=\frac{Q(0.75)-2 Q(0.5)+Q(0.25)}{Q(0.75)-Q(0.25)}
$$

And the Moors [21] kurtosis based on octiles:

$$
K U R(M)=\frac{Q(0.875)-Q(0.625)+Q(0.375)-Q(0.125)}{Q(0.75)-Q(0.25)},
$$

where $Q($.$) stands for the quantile function define in (15).$ 
Here, we followed Alizadeh et al. [1] and Handique and Chakraborty [14] method of limiting behaviour, we have

The asymptotic of Equations (4) and (8) as $x \rightarrow \infty$ are given by

$$
\begin{aligned}
& \lim _{x \rightarrow \infty} f(x)=\lim _{x \rightarrow \infty} \frac{c \lambda \gamma \theta \infty^{\theta-1} e^{-\gamma \infty^{\theta}}\left(1-e^{-\gamma_{\infty} \theta}\right)^{c-1}}{B(\alpha, \beta)}\left(1-\left(1-e^{-\gamma_{\infty} \theta}\right)^{c}\right)^{\lambda \beta-1} \\
& \times\left(1-\left(1-\left(1-e^{-\gamma \infty^{\theta}}\right)^{c}\right)^{\lambda}\right)^{\alpha-1}=\lim _{x \rightarrow \infty} f(x)=0, \quad \text { as } x \rightarrow \infty, \\
& \lim _{x \rightarrow \infty} h z(x)=\lim _{x \rightarrow \infty} \frac{c \lambda \gamma \theta \infty^{\theta-1} e^{-\gamma_{\infty} \theta}\left(1-e^{-\gamma_{\infty} \theta}\right)^{c-1}}{\left.\left.B(\alpha, \beta)-I_{\left[1-\left(1-e^{-\gamma \infty}{ }^{\theta}\right.\right.}\right)^{c}\right]^{\lambda}(\alpha, \beta)}\left(1-\left(1-e^{-\gamma_{\infty} \theta}\right)^{c}\right)^{\lambda \beta-1} \\
& \times\left(1-\left(1-\left(1-e^{-\gamma_{\infty} \theta}\right)^{c}\right)^{\lambda}\right)^{\alpha-1}=\lim _{x \rightarrow \infty} h z(x)=0, \quad \text { as } x \rightarrow \infty .
\end{aligned}
$$

The asymptotic of Equations (4) or (5) and (10) as $x \rightarrow 0$ are given by

$$
\begin{aligned}
\lim _{f(x) \rightarrow 0}= & \lim _{f(x) \rightarrow 0} \frac{c \lambda \gamma \theta 0^{\theta-1} e^{-\gamma 0^{\theta}}\left(1-e^{-\gamma 0^{\theta}}\right)^{c-1}}{B(\alpha, \beta)}\left(1-\left(1-e^{-\gamma 0^{\theta}}\right)^{c}\right)^{\lambda \beta-1} \\
& \times\left(1-\left(1-\left(1-e^{-\gamma 0^{\theta}}\right)^{c}\right)^{\lambda}\right)^{\alpha-1}=\lim _{x \rightarrow 0} f(x)=0, \quad \text { as } f(x) \rightarrow 0, \\
\lim _{f(x) \rightarrow 0} h z(x) & \left.=\lim _{f(x) \rightarrow 0} \frac{c \lambda \gamma \theta 0^{\theta-1} e^{-\gamma 0^{\theta}}\left(1-e^{-\gamma 0^{\theta}}\right)^{c-1}}{B(\alpha, \beta)-I_{[}}\left(1-\left(1-e^{-\gamma 0^{\theta}}\right)^{c}\right]^{\lambda(\alpha, \beta)}\left(1-e^{-\gamma 0^{\theta}}\right)^{c}\right)^{\lambda \beta-1} \\
& \times\left(1-\left(1-\left(1-e^{-\gamma 0^{\theta}}\right)^{c}\right)^{\lambda}\right)^{\alpha-1}=\lim _{f(x) \rightarrow 0} h z(x)=0, \quad \text { as } f(x) \rightarrow 0 .
\end{aligned}
$$




\section{Estimation of Parameter}

The parameters of GBEW distribution is estimated through maximum likelihood. Here, let $\theta$ be a vector of parameters, Cordeiro and de Castro [8] gave the log-likelihood for $\theta=(\alpha, \beta, c, \lambda, \xi)$, where $\xi=(\gamma, \theta)$ as

$$
\begin{aligned}
l(\theta)= & n \log (c \lambda)+\sum_{i=1}^{n} \log [f(x ; \xi)]-n \log [B(\alpha, \beta)] \\
& +(c-1) \sum_{i=1}^{n} \log [F(x ; \xi)]+(\lambda \beta-1) \sum_{i=1}^{n} \log \left[1-F(x ; \xi)^{c}\right] \\
& +(\alpha-1) \sum_{i=1}^{n} \log \left[1-\left[1-F(x ; \xi)^{c}\right]^{\lambda}\right]
\end{aligned}
$$

where $f(x ; \xi)=\gamma \theta x^{\theta-1} e^{-\gamma x^{\theta}}$ and $F(x ; \xi)=\left[1-e^{-\gamma x^{\theta}}\right]$.

Taking the partial derivatives of the log-likelihood function with respect to $\alpha, \beta, c, \lambda, \xi$ components can be obtained as follows:

$$
\begin{gathered}
\frac{\partial l(\theta)}{\partial \alpha}=-n \varphi(\alpha)+n \varphi(\alpha+\beta)+\sum_{i=1}^{n} \log \left[1-\left[1-F(x ; \xi)^{c}\right]^{\lambda}\right] \\
\frac{\partial l(\theta)}{\partial \beta}=-n \varphi(\beta)+n \varphi(\alpha+\beta)+\lambda \sum_{i=1}^{n} \log \left[1-F(x ; \xi)^{c}\right], \\
\frac{\partial l(\theta)}{\partial c}=\frac{n}{c}+\sum_{i=1}^{n} \log [F(x ; \xi)]+(1-\lambda \beta) \sum_{i=1}^{n} \frac{F(x ; \xi)^{c} \log [F(x ; \xi)]}{1-F(x ; \xi)} \\
+(\alpha-1) \sum_{i=1}^{n} \frac{\lambda\left[1-F(x ; \xi)^{c}\right]^{\lambda-1} \log [F(x ; \xi)]}{1-\left[1-F(x ; \xi)^{c}\right]^{\lambda}}
\end{gathered}
$$


$\frac{\partial l(\theta)}{\partial \lambda}=\frac{n}{\lambda}+\beta \sum_{i=1}^{n} \log \left[1-F(x ; \xi)^{c}\right]+(1-\alpha) \sum_{i=1}^{n} \frac{\left[1-F(x ; \xi)^{c}\right]^{\lambda} \log \left[F(x ; \xi)^{c}\right]}{1-\left[1-F(x ; \xi)^{c}\right]^{\lambda}}$

$$
\begin{aligned}
\frac{\partial l(\theta)}{\partial \gamma}= & \frac{n \theta}{\gamma}-\frac{\theta \gamma^{\theta-1} f(x ; \xi)}{f(x ; \xi)}+(c-1) \sum_{i=1}^{n} \frac{\theta \gamma^{\theta-1} F(x ; \xi)}{F(x ; \xi)} \\
& +(1-\lambda \beta) \sum_{i=1}^{n} \frac{c F(x ; \xi)^{c-1} \theta \gamma^{\theta-1} F(x ; \xi)}{1-F(x ; \xi)^{c}} \\
& +(\alpha-1) \sum_{i=1}^{n} \frac{\lambda\left[1-F(x ; \xi)^{c}\right]^{\lambda-1} c F(x ; \xi)^{c-1} \theta \gamma^{\theta-1} F(x ; \xi)}{1-\left[1-F(x ; \xi)^{c}\right]^{\lambda}} . \\
\frac{\partial l(\theta)}{\partial \theta}= & \frac{n}{\theta}+\gamma^{\theta} \sum_{i=1}^{n} \frac{\gamma^{\theta} F(x ; \xi)}{F(x ; \xi)}+(c-1) \gamma^{\theta} \sum_{i=1}^{n} \frac{\gamma^{\theta} F(x ; \xi)}{F(x ; \xi)} \\
& +(1-\lambda \beta) \gamma^{\theta} \sum_{i=1}^{n} \frac{c F(x ; \xi)^{c-1} F(x ; \xi)}{1-F(x ; \xi)^{c}} \\
& +(\alpha-1) \gamma^{\theta} \sum_{i=1}^{n} \frac{\lambda\left[1-F(x ; \xi)^{c}\right]^{\lambda-1} c F(x ; \xi)^{c-1} \gamma^{\theta} F(x ; \xi)}{1-\left[1-F(x ; \xi)^{c}\right]^{\lambda}}
\end{aligned}
$$

Then, the asymptotic variance-covariance matrix for mles of the parameters of GBEW distribution is derived by taking second partial derivatives as follows:

$$
\begin{aligned}
& \frac{\partial^{2} l(\theta)}{\partial \alpha^{2}}=-n \psi^{\prime}(\alpha)-n \psi^{\prime}(\alpha+\beta), \\
& \frac{\partial^{2} l(\theta)}{\partial \beta^{2}}=-n \psi^{\prime}(\beta)-n \psi^{\prime}(\alpha+\beta),
\end{aligned}
$$




$$
\begin{aligned}
\frac{\partial^{2} l(\theta)}{\partial c^{2}}= & \frac{n}{c^{2}}+(1-\lambda \beta) \sum_{i=1}^{n} \\
& \times\left[\frac{\left[1-e^{-\gamma x_{i}^{\theta}}\right]^{2 c} \log \left(1-e^{-\gamma x_{i}^{\theta}}\right)^{2}}{\left[1-\left(1-e^{-\gamma x_{i}^{\theta}}\right)^{c}\right]^{2}}+\frac{\left(1-e^{-\gamma x_{i}^{\theta}}\right)^{c} \log \left(1-e^{-\gamma x_{i}^{\theta}}\right)^{2}}{1-\left(1-e^{-\gamma x_{i}^{\theta}}\right)^{c}}\right] \\
& +(1-\alpha) \sum_{i=1}^{n} \frac{\lambda^{2}\left[1-\left(1-e^{-\gamma x_{i}^{\theta}}\right)^{c}\right]^{2(\lambda-1)}\left(1-e^{-\gamma x_{i}^{\theta}}\right)^{2 c} \log \left[\left(1-e^{-\gamma x_{i}^{\theta}}\right)\right]^{2}}{\left.\left.\left[1-\left(1-e^{-\gamma x_{i}^{\theta}}\right)^{c}\right)^{\lambda}\right]^{2}\right]^{2}} \\
& +(1-\alpha) \sum_{i=1}^{n} \frac{\lambda(\lambda-1)\left[1-\left(1-e^{-\gamma x_{i}^{\theta}}\right)^{c}\right]^{\lambda-2}\left(1-e^{-\gamma x_{i}^{\theta}}\right)^{2 c} \log \left[\left(1-e^{-\gamma x_{i}^{\theta}}\right)\right]^{2}}{1-\left(1-\left(1-e^{-\gamma x_{i}^{\theta}}\right)^{c}\right)^{\lambda}} \\
& -(1-\alpha) \sum_{i=1}^{n} \frac{\left.\lambda\left[1-\left(1-e^{-\gamma x_{i}^{\theta}}\right)^{c}\right]^{\lambda-1}\left(1-e^{-\gamma x_{i}^{\theta}}\right)^{c} \log \left[\left(1-e^{-\gamma x_{i}^{\theta}}\right)\right]^{c}\right)^{\lambda}}{n^{2}}
\end{aligned}
$$




$$
\begin{aligned}
& \frac{\partial^{2} l(\theta)}{\partial \lambda^{2}}=\frac{n}{\lambda}+(1-\alpha) \sum_{i=1}^{n} \frac{\left[1-\left(1-e^{-\gamma x_{i}^{\theta}}\right)^{c}\right]^{2 \lambda} \log \left[1-\left(1-e^{-\gamma x_{i}^{\theta}}\right)^{c}\right]^{2}}{\left[1-\left(1-\left(1-e^{-\gamma x_{i}^{\theta}}\right)^{c}\right)^{\lambda}\right]^{2}} \\
& +(1-\alpha) \sum_{i=1}^{n} \frac{\left[1-\left(1-e^{-\gamma x_{i}^{\theta}}\right)^{c}\right]^{\lambda} \log \left[1-\left(1-e^{-\gamma x_{i}^{\theta}}\right)^{c}\right]^{2}}{1-\left[1-1-\left(1-e^{-\gamma x_{i}^{\theta}}\right)^{c}\right]^{2}}, \\
& \frac{\partial^{2} l(\theta)}{\partial \gamma^{2}}=\frac{n}{\gamma^{2}}+(c-1) \sum_{i=1}^{n}\left(\frac{e^{-2 \gamma x_{i}^{\theta}} \theta x_{i}^{\theta-1}}{\left[\left(1-e^{-\gamma x_{i}^{\theta}}\right)\right]^{2}}-\frac{e^{-\gamma x_{i}^{\theta}} \theta x_{i}^{\theta-1}}{1-e^{-\gamma x_{i}^{\theta}}}\right) \\
& +(1-\lambda \beta) \sum_{i=1}^{n} \frac{c^{2}\left(1-e^{-\gamma x_{i}^{\theta}}\right)^{2(c-1)} e^{-\gamma x_{i}^{\theta}} \theta x_{i}^{\theta-1}}{\left[1-1-\left(1-e^{-\gamma x_{i}^{\theta}}\right)^{c}\right]^{2}} \\
& +(1-\lambda \beta) \sum_{i=1}^{n} \frac{c(c-1)\left(1-e^{-\gamma x_{i}^{\theta}}\right)^{(c-2)} e^{-\gamma x_{i}^{\theta}} \theta x_{i}^{\theta-1}}{1-1-\left(1-e^{-\gamma x_{i}^{\theta}}\right)^{c}} \\
& -(1-\lambda \beta) \sum_{i=1}^{n} \frac{c\left(1-e^{-\gamma x_{i}^{\theta}}\right)^{(c-1)} e^{-\gamma x_{i}^{\theta}} \theta x_{i}^{\theta-1}}{1-1-\left(1-e^{-\gamma x_{i}^{\theta}}\right)^{c}}
\end{aligned}
$$




$$
\begin{aligned}
& +(1-\alpha) \sum_{i=1}^{n} \frac{c^{2} \lambda^{2}\left[1-\left(1-e^{-\gamma x_{i}^{\theta}}\right)^{c}\right]^{2(\lambda-1)}\left(1-e^{-\gamma x_{i}^{\theta}}\right)^{2(c-1)} e^{-\gamma x_{i}^{\theta}} \theta x_{i}^{\theta-1}}{\left[1-\left(1-\left(1-e^{-\gamma x_{i}^{\theta}}\right)^{c}\right)^{\lambda}\right]^{2}} \\
& +(1-\alpha) \sum_{i=1}^{n} \frac{c^{2} \lambda(\lambda-1)\left[1-\left(1-e^{-\gamma x_{i}^{\theta}}\right)^{c}\right]^{\lambda-2}\left(1-e^{-\gamma x_{i}^{\theta}}\right)^{2(c-1)} e^{-\gamma x_{i}^{\theta}} \theta x_{i}^{\theta-1}}{1-\left(1-\left(1-e^{-\gamma x_{i}^{\theta}}\right)^{c}\right)^{\lambda}} \\
& -(1-\alpha) \sum_{i=1}^{n} \frac{c(c-1) \lambda\left[1-\left(1-e^{-\gamma x_{i}^{\theta}}\right)^{c}\right]^{\lambda-1}\left(1-e^{-\gamma x_{i}^{\theta}}\right)^{c-2} e^{-\gamma x_{i}^{\theta}} \theta x_{i}^{\theta-1}}{1-\left(1-\left(1-e^{-\gamma x_{i}^{\theta}}\right)^{c}\right)^{\lambda}} \\
& +(1-\alpha) \sum_{i=1}^{n} \frac{c \lambda\left[1-\left(1-e^{-\gamma x_{i}^{\theta}}\right)^{c}\right]^{\lambda-1}\left(1-e^{-\gamma x_{i}^{\theta}}\right)^{c-1} e^{-\gamma x_{i}^{\theta}} \theta x_{i}^{\theta-1}}{1-\left(1-\left(1-e^{-\gamma x_{i}^{\theta}}\right)^{c}\right)^{\lambda}} \\
& \frac{\partial^{2} l(\theta)}{\partial \theta^{2}}=\frac{n}{\theta^{2}}+\frac{e^{-\theta x_{i}^{\theta-1}} e^{-\gamma x_{i}^{\theta}} \theta x_{i}^{\theta-1}}{e^{-\gamma x_{i}^{\theta}}}+(c-1) \sum_{i=1}^{n} \frac{e^{-2 x_{i}^{\theta}} \theta x_{i}^{\theta-1}}{\left(1-e^{-\gamma x_{i}^{\theta}}\right)}-\frac{e^{-x_{i}^{\theta}} \theta x_{i}^{\theta-1}}{1-e^{-\gamma x_{i}^{\theta}}} \\
& +(1-\lambda \beta) \sum_{i=1}^{n} \frac{c^{2}\left(1-e^{-\gamma x_{i}^{\theta}}\right)^{2(c-1)} e^{-x_{i}^{\theta}} \theta x_{i}^{\theta-1}}{\left[1-1-\left(1-e^{-\gamma x_{i}^{\theta}}\right)^{c}\right]^{2}}
\end{aligned}
$$




$$
\begin{aligned}
& +(1-\lambda \beta) \sum_{i=1}^{n} \frac{c(c-1)\left(1-e^{\left.-\gamma x_{i}^{\theta}\right)^{(c-2)} e^{-x_{i}^{\theta}} \theta^{2} x_{i}^{\theta-2}}\right.}{1-\left(1-e^{-\gamma x_{i}^{\theta}}\right)^{c}} \\
& -(1-\lambda \beta) \sum_{i=1}^{n} \frac{c\left(1-e^{\left.-\gamma x_{i}^{\theta}\right)^{(c-1)}} e^{-x_{i}^{\theta}} \theta^{2} x_{i}^{\theta-2}\right.}{1-\left(1-e^{-\gamma x_{i}^{\theta}}\right)^{c}} \\
& +(1-\alpha) \sum_{i=1}^{n} \frac{c^{2} \lambda^{2}\left[1-\left(1-e^{-\gamma x_{i}^{\theta}}\right)^{c}\right]^{2(\lambda-1)}\left(1-e^{-\gamma x_{i}^{\theta}}\right)^{2(c-1)} e^{-x_{i}^{\theta}} \theta^{2} x_{i}^{\theta-2}}{\left[1-\left(1-\left(1-e^{-\gamma x_{i}^{\theta}}\right)^{c}\right)^{\lambda}\right]^{2}} \\
& +(1-\alpha) \sum_{i=1}^{n} \frac{c^{2} \lambda(\lambda-1)\left[1-\left(1-e^{-\gamma x_{i}^{\theta}}\right)^{c} \sum_{i=1}^{n} \frac{\partial^{2} l(\theta)}{\partial \alpha \partial \beta}=n \psi^{\prime}(\alpha+\beta),\left(1-e^{-\gamma x_{i}^{\theta}}\right)^{2(c-1)} e^{-x_{i}^{\theta}} \theta^{2} x_{i}^{\theta-2}\right.}{\left.\left.c(c-1) \lambda\left[1-\left(1-e^{-\gamma x_{i}^{\theta}}\right)^{c}\right]^{\lambda-1}\left(1-e^{\theta}\right)^{\lambda}\right)^{-\gamma x_{i}^{\theta}}\right)^{c-2} e^{-x_{i}^{\theta} \theta^{2} x_{i}^{\theta-2}}}
\end{aligned}
$$




$$
\begin{aligned}
& \frac{\partial^{2} l(\theta)}{\partial \alpha \partial c}=\sum_{i=1}^{n} \frac{\lambda\left[1-\left(1-e^{-\gamma x_{i}^{\theta}}\right)^{c}\right]^{\lambda-1}\left(1-e^{-\gamma x_{i}^{\theta}}\right)^{c} \log \left[1-\left(1-e^{-\gamma x_{i}^{\theta}}\right)\right]}{1-\left[1-\left(1-e^{-\gamma x_{i}^{\theta}}\right)^{c}\right]^{\lambda}}, \\
& \frac{\partial^{2} l(\theta)}{\partial \alpha \partial \lambda}=\sum_{i=1}^{n}-\frac{\left[1-\left(1-e^{-\gamma x_{i}^{\theta}}\right)^{c}\right]^{\lambda-1} \log \left[1-\left(1-e^{-\gamma x_{i}^{\theta}}\right)^{c}\right]}{1-\left[1-\left(1-e^{-\gamma x_{i}^{\theta}}\right)^{c}\right]^{\lambda}}, \\
& \frac{\partial^{2} l(\theta)}{\partial \alpha \partial \gamma}=\sum_{i=1}^{n} \frac{c \lambda\left[1-\left(1-e^{-\gamma x_{i}^{\theta}}\right)^{c}\right]^{\lambda}\left(1-e^{-\gamma x_{i}^{\theta}}\right)^{c} e^{-\gamma x_{i}^{\theta}} x_{i}^{\theta}}{1-\left[1-\left(1-e^{-\gamma x_{i}^{\theta}}\right)^{c}\right]^{\lambda}} \\
& \frac{\partial^{2} l(\theta)}{\partial \alpha \partial \theta}=\sum_{i=1}^{n} \frac{c \lambda\left[1-\left(1-e^{-\gamma x_{i}^{\theta}}\right)^{c}\right]^{\lambda}\left(1-e^{-\gamma x_{i}^{\theta}}\right)^{c} e^{-\gamma x_{i}^{\theta}}}{1-\left[1-\left(1-e^{-\gamma x_{i}^{\theta}}\right)^{c}\right]^{\lambda}}, \\
& \frac{\partial^{2} l(\theta)}{\partial \beta \partial \alpha}=n \psi^{\prime}(\alpha+\beta), \quad \frac{\partial^{2} l(\theta)}{\partial \beta \partial c}=\lambda \sum_{i=1}^{n}-\frac{\left(1-e^{-\gamma x_{i}^{\theta}}\right)^{c} \log \left(1-e^{-\gamma x_{i}^{\theta}}\right)}{1-\left(1-e^{-\gamma x_{i}^{\theta}}\right)^{c}}, \\
& \frac{\partial^{2} l(\theta)}{\partial \beta \partial \lambda}=\sum_{i=1}^{n} \log \left[1-\left(1-e^{-\gamma x_{i}^{\theta}}\right)^{c}\right], \frac{\partial^{2} l(\theta)}{\partial \beta \partial \gamma}=\lambda \sum_{i=1}^{n}-\frac{c \gamma\left(1-e^{-\gamma x_{i}^{\theta}}\right)^{c-1} e^{-\gamma x_{i}^{\theta}}}{1-\left(1-e^{-\gamma x_{i}^{\theta}}\right)^{c}},
\end{aligned}
$$




$$
\begin{aligned}
& \frac{\partial^{2} l(\theta)}{\partial \beta \partial \lambda}=-\beta \sum_{i=1}^{n}-\frac{\left(1-e^{-\gamma x_{i}^{\theta}}\right)^{c} \log \left(1-e^{-\gamma x_{i}^{\theta}}\right)}{1-\left(1-e^{-\gamma x_{i}^{\theta}}\right)} \\
& +(\alpha-1) \sum_{i=1}^{n} \frac{\left[1-\left(1-e^{-\gamma x_{i}^{\theta}}\right)^{c}\right]^{\lambda-1}\left(1-e^{-\gamma x_{i}^{\theta}}\right)^{c} \log \left(1-e^{-\gamma x_{i}^{\theta}}\right)}{1-\left[1-\left(1-e^{-\gamma x_{i}^{\theta}}\right)^{c}\right]^{\lambda}} \\
& +\lambda(\alpha-1) \sum_{i=1}^{n} \frac{\left[1-\left(1-e^{-\gamma x_{i}^{\theta}}\right)^{c}\right]^{2 \lambda-1}\left(1-e^{-\gamma x_{i}^{\theta}}\right)^{c} \log \left(1-e^{-\gamma x_{i}^{\theta}}\right) \log \left[1-\left(1-e^{-\gamma x_{i}^{\theta}}\right)^{c}\right]}{\left[1-\left[1-\left(1-e^{-\gamma x_{i}^{\theta}}\right)^{c}\right]^{\lambda}\right]^{2}} \\
& +\lambda(\alpha-1) \sum_{i=1}^{n} \frac{\left[1-\left(1-e^{-\gamma x_{i}^{\theta}}\right)^{c}\right]^{\lambda-1}\left(1-e^{-\gamma x_{i}^{\theta}}\right)^{c} \log \left(1-e^{-\gamma x_{i}^{\theta}}\right) \log \left[1-\left(1-e^{-\gamma x_{i}^{\theta}}\right)^{c}\right]}{1-\left[1-\left(1-e^{-\gamma x_{i}^{\theta}}\right)^{c}\right]^{\lambda}}, \\
& \frac{\partial^{2} l(\theta)}{\partial c \partial \gamma}=\sum_{i=1}^{n} \frac{\gamma e^{-\gamma x_{i}^{\theta}} x_{i}^{\theta}}{1-e^{-\gamma x_{i}^{\theta}}}+(1-\beta \lambda) \sum_{i=1}^{n} \frac{c\left(1-e^{-\gamma x_{i}^{\theta}}\right)^{c-1} e^{-\gamma x_{i}^{\theta}} x_{i}^{\theta}}{1-\left(1-e^{-\gamma x_{i}^{\theta}}\right)^{c}} \\
& +(1-\beta \lambda) \sum_{i=1}^{n} \frac{c\left(1-e^{-\gamma x_{i}^{\theta}}\right)^{2 c-1} e^{-\gamma x_{i}^{\theta}} \log \left(1-e^{-\gamma x_{i}^{\theta}}\right) x_{i}^{\theta}}{\left[1-\left(1-e^{-\gamma x_{i}^{\theta}}\right)^{c}\right]^{2}}
\end{aligned}
$$


GENERALIZED BETA-EXPONENTIAL WEIBULL ...

21

$$
\begin{aligned}
& +(1-\beta \lambda) \sum_{i=1}^{n} \frac{c\left(1-e^{-\gamma x_{i}^{\theta}}\right)^{c-1} e^{-\gamma x_{i}^{\theta}} \log \left(1-e^{-\gamma x_{i}^{\theta}}\right) x_{i}^{\theta}}{1-\left(1-e^{-\gamma x_{i}^{\theta}}\right)^{c}} \\
& +(\alpha-1) \sum_{i=1}^{n} \frac{c \lambda\left[1-\left(1-e^{-\gamma x_{i}^{\theta}}\right)^{c}\right]^{\lambda-1}\left(1-e^{-\gamma x_{i}^{\theta}}\right)^{c-1} e^{-\gamma x_{i}^{\theta}} x_{i}^{\theta}}{1-\left[1-\left(1-e^{-\gamma x_{i}^{\theta}}\right)^{c}\right]^{\lambda}} \\
& +(\alpha-1) \sum_{i=1}^{n} \frac{c \lambda^{2}\left[1-\left(1-e^{-\gamma x_{i}^{\theta}}\right)^{c}\right]^{2(\lambda-1)}\left(1-e^{-\gamma x_{i}^{\theta}}\right)^{2 c-1} e^{-\gamma x_{i}^{\theta}} x_{i}^{\theta} \log \left(1-e^{-\gamma x_{i}^{\theta}}\right)}{\left[1-\left[1-\left(1-e^{-\gamma x_{i}^{\theta}}\right)^{c}\right]^{\lambda}\right]^{2}} \\
& +(\alpha-1) \sum_{i=1}^{n} \frac{c \lambda(\lambda-1)\left[1-\left(1-e^{-\gamma x_{i}^{\theta}}\right)^{c}\right]^{\lambda-2}\left(1-e^{-\gamma x_{i}^{\theta}}\right)^{2 c-1} e^{-\gamma x_{i}^{\theta}} x_{i}^{\theta} \log \left(1-e^{-\gamma x_{i}^{\theta}}\right)}{1-\left[1-\left(1-e^{-\gamma x_{i}^{\theta}}\right)^{c}\right]^{\lambda}} \\
& +(\alpha-1) \sum_{i=1}^{n} \frac{c \lambda\left[1-\left(1-e^{-\gamma x_{i}^{\theta}}\right)^{c}\right]^{\lambda-1}\left(1-e^{-\gamma x_{i}^{\theta}}\right)^{c-1} e^{-\gamma x_{i}^{\theta}} x_{i}^{\theta} \log \left(1-e^{-\gamma x_{i}^{\theta}}\right)}{1-\left[1-\left(1-e^{-\gamma x_{i}^{\theta}}\right)^{c}\right]^{\lambda}}
\end{aligned}
$$




$$
\begin{gathered}
\frac{\partial^{2} l(\theta)}{\partial c \partial \theta}=\sum_{i=1}^{n} \frac{\gamma e^{-\gamma x_{i}^{\theta}}}{1-e^{-\gamma x_{i}^{\theta}}}+(1-\beta \lambda) \sum_{i=1}^{n} \frac{c\left(1-e^{-\gamma x_{i}^{\theta}}\right)^{c-1} e^{-\gamma x_{i}^{\theta}}}{1-\left(1-e^{-\gamma x_{i}^{\theta}}\right)^{c}}, \\
\frac{\partial^{2} l(\theta)}{\partial \lambda \partial \gamma}=-\beta \sum_{i=1}^{n} \frac{c\left(1-e^{-\gamma x_{i}^{\theta}}\right)^{c-1} e^{-\gamma x_{i}^{\theta}} x_{i}^{\theta}}{1-\left(1-e^{-\gamma x_{i}^{\theta}}\right)^{c}}, \frac{\partial^{2} l(\theta)}{\partial \lambda \partial \theta}=-\beta \sum_{i=1}^{n} \frac{c\left(1-e^{-\gamma x_{i}^{\theta}}\right)^{c-1} e^{-\gamma x_{i}^{\theta}}}{1-\left(1-e^{-\gamma x_{i}^{\theta}}\right)^{c}},
\end{gathered}
$$

and lastly,

$$
\begin{aligned}
& \frac{\partial^{2} l(\theta)}{\partial \gamma \partial \theta}= \frac{\gamma e^{-\gamma x_{i}^{\theta}}}{1-e^{-\gamma x_{i}^{\theta}}}+(c-1) \sum_{i=1}^{n} \frac{\gamma e^{-\gamma x_{i}^{\theta}} \log \left[1-\left(1-e^{-\gamma x_{i}^{\theta}}\right)\right] x_{i}^{\theta}}{1-\left(1-e^{-\gamma x_{i}^{\theta}}\right)^{c}} \\
&+(\lambda \beta-1) \sum_{i=1}^{n} \frac{\left(\gamma e^{-\gamma x_{i}^{\theta}}\right)^{c-1} \log \left[1-\left(1-e^{-\gamma x_{i}^{\theta}}\right)\right]^{2} x_{i}^{\theta}}{\left[1-\left(1-e^{-\gamma x_{i}^{\theta}}\right)^{c}\right]^{2}} \\
&+(\alpha-1) \sum_{i=1}^{n} \frac{\gamma\left[1-\left(1-e^{-\gamma x_{i}^{\theta}}\right)^{c}\right]^{\lambda-1}\left[\log \left[1-\left(1-\left(1-e^{-\gamma x_{i}^{\theta}}\right)^{c}\right)\right]^{\lambda} x_{i}^{\theta}\right.}{\left[1-\left(1-\left(1-e^{-\gamma x_{i}^{\theta}}\right)^{c}\right)^{\lambda}\right]^{2}} .
\end{aligned}
$$

Hence, the asymptotic variance covariance matrix for mles of the parameters of GBEW $(\alpha, \beta, c, \lambda, \gamma, \theta)$ distribution is estimated as given below: 


$l^{-1}(\hat{\theta})=$
$\left[\begin{array}{cccccc}v(\hat{\alpha}) & c v(\hat{\alpha}, \hat{\beta}) & c v(\hat{\alpha}, \hat{c}) & c v(\hat{\alpha}, \hat{\lambda}) & c v(\hat{\alpha}, \hat{\gamma}) & c v(\hat{\alpha}, \hat{\theta}) \\ c v(\hat{\beta}, \hat{\alpha}) & v(\hat{\beta}) & c v(\hat{\beta}, \hat{c}) & c v(\hat{\beta}, \hat{\lambda}) & c v(\hat{\beta}, \hat{\gamma}) & c v(\hat{\beta}, \hat{\theta}) \\ c v(\hat{c}, \hat{\alpha}) & c v(\hat{c}, \hat{\beta}) & v(\hat{c}) & c v(\hat{c}, \hat{\lambda}) & c v(\hat{c}, \hat{\gamma}) & c v(\hat{c}, \hat{\theta}) \\ c v(\hat{\lambda}, \hat{\alpha}) & c v(\hat{\lambda}, \hat{\beta}) & c v(\hat{\lambda}, \hat{c}) & v(\hat{\lambda}) & c v(\hat{\lambda}, \hat{\gamma}) & c v(\hat{\lambda}, \hat{\theta}) \\ c v(\hat{\gamma}, \hat{\alpha}) & c v(\hat{\gamma}, \hat{\beta}) & c v(\hat{\gamma}, \hat{c}) & c v(\hat{\gamma}, \hat{\lambda}) & v(\hat{\gamma}) & c v(\hat{\gamma}, \hat{\theta}) \\ c v(\hat{\theta}, \hat{\alpha}) & c v(\hat{\theta}, \hat{\beta}) & c v(\hat{\theta}, \hat{c}) & c v(\hat{\theta}, \hat{\lambda}) & c v(\hat{\theta}, \hat{\gamma}) & v(\hat{\theta})\end{array}\right]$.

Therefore, the elements of the information matrix $l^{-1}(\hat{\theta})=\left[\frac{\partial^{2} l(\theta)}{\partial \theta_{i} \partial \theta_{j}}\right]_{\theta=\hat{\theta}}$ have been derived in the above using second partial derivatives.

\subsection{Analysis of data}

\subsubsection{Data set 1}

The data set 1 is on the strengths of $1.5 \mathrm{~cm}$ glass fibres. The data was originally obtained by workers at the UK National Physical Laboratory and it has been used by Smith and Naylor [31] and Bourguignon et al. [6]. In the first application, GBEW is compared with other generalized models such as the Beta Weibull (BW) (Famoye et al. [11] and Lee et al. [16]), Beta Exponential (BE) (Nadarajah and Kotz [22]), Exponentiated Exponential (EE) (Gupta and Kundu [12]), Exponential Weibull (EW) (Salem and Abo-Kasem [30] and Choudhury [7]), Exponentiated Rayleigh (ER) (Mahmoud and Ghazal [18]), Exponentiated Exponential Rayleigh (EER), and Beta Rayleigh (BR).

The values of the maximum likelihood estimates (MLEs) and their corresponding standard errors (in parentheses) of the model parameters for the first data set are given in Table 2. Then, in Table 3, we compare 
the fits of the GBEW, BW, BE, EE, EW, ER, EER, and BR distributions. The values in Table 3 now indicate that the generalized beta exponential Weibull (GBEW) model has the lowest values for the log-likelihood, AIC, CAIC, HQIC, and BIC statistics (for the first data set) among the fitted models. Hence, the GBEW model is the best fitted model among other competing models. While, Figure 2 are the plots of the observed Histogram, estimated pdfs and cdfs for the distributions for data set 1 (strengths of $1.5 \mathrm{~cm}$ glass fibres data).

Table 2. MLEs of the parameters and their standard errors (in parentheses) for strengths of $1.5 \mathrm{~cm}$ glass fibres data

\begin{tabular}{|c|c|c|c|c|c|c|}
\hline Model/Parameter & $\hat{a}$ & $\hat{b}$ & $\hat{c}$ & $\hat{\gamma}$ & $\hat{\hat{\lambda}}$ & $\hat{\theta}$ \\
\hline \multirow[t]{2}{*}{$\operatorname{GBEW}(\alpha, \beta, c, \gamma, \lambda, \theta)$} & 0.0045 & 4.9183 & 1.8114 & 2.8749 & 2.8671 & 3.2318 \\
\hline & $(0.0008)$ & $(0.0013)$ & $(3.1050)$ & (3.0930) & $(3.0920)$ & (3.0930) \\
\hline \multirow[t]{2}{*}{$\mathrm{BW}(\alpha, \beta, 1, \gamma, 1, \theta)$} & 0.0144 & 4.9660 & 1 & 2.9830 & 1 & 3.0770 \\
\hline & $(0.0017)$ & $(1.0021)$ & & $(0.0220)$ & & $(0.0100)$ \\
\hline \multirow[t]{2}{*}{$\mathrm{BE}(\alpha, \beta, 1, \gamma, 1,1)$} & 0.0119 & 4.9401 & 1 & 2.9352 & 1 & 1 \\
\hline & $(0.2011)$ & $(1.2390)$ & & $(0.3422)$ & & \\
\hline \multirow[t]{2}{*}{$\operatorname{EE}(1,1, c, \gamma, \lambda, 1)$} & 1 & 1 & 0.5653 & 2.5570 & 0.5310 & 1 \\
\hline & & & $(0.1144)$ & $(0.0004)$ & $(0.0025)$ & \\
\hline \multirow[t]{2}{*}{$\mathrm{EW}(\alpha, 1, c, \gamma, 1, \theta)$} & 0.0185 & 1 & 1.9110 & 2.9950 & 1 & 3.0300 \\
\hline & $(0.0043)$ & & $(0.1900)$ & $(0.0000)$ & & $(0.0000)$ \\
\hline \multirow[t]{2}{*}{$\mathrm{ER}(1,1,1, \gamma, 1,2)$} & 1 & 1 & 1 & 2.9310 & 1 & 2 \\
\hline & & & & $(0.0000)$ & & \\
\hline \multirow[t]{2}{*}{$\operatorname{EER}(1,1, c, \gamma, \lambda, 2)$} & 1 & 1 & 1.9050 & 2.8750 & 2.8960 & 2 \\
\hline & & & $(0.0000)$ & $(0.0000)$ & $(0.0000)$ & \\
\hline \multirow[t]{2}{*}{$\mathrm{BR}(\alpha, \beta, 1, \gamma, 1,2)$} & 0.0028 & 4.9520 & 1 & 2.9670 & 1 & 2 \\
\hline & (NA) & (NA) & & $(0.0001)$ & & \\
\hline
\end{tabular}


Table 3. Goodness-of-fit statistics for strengths of $1.5 \mathrm{~cm}$ glass fibres data

\begin{tabular}{lccccc}
\hline Model & -2 Loglik & AIC & CAIC & HQIC & BIC \\
\hline GBEW & $\mathbf{8 9 0 . 2 2 1 7}$ & $\mathbf{1 7 9 2 . 4 4 3}$ & $\mathbf{1 7 9 3 . 9 4 3}$ & $\mathbf{1 7 9 7 . 5 0 1}$ & $\mathbf{1 8 0 5 . 3 0 2}$ \\
BW & 909.2020 & 1826.401 & 1827.094 & 1829.776 & 1834.977 \\
EW & 912.2010 & 1832.402 & 1833.092 & 1835.774 & 1840.975 \\
EER & 914.3140 & 1834.628 & 1835.035 & 1837.157 & 1841.057 \\
BR & 915.1140 & 1836.228 & 1836.635 & 1838.757 & 1842.657 \\
BE & 916.1310 & 1838.262 & 1838.669 & 1840.791 & 1844.691 \\
EE & 918.1010 & 1842.202 & 1842.609 & 1844.731 & 1848.631 \\
ER & 972.0010 & 1946.002 & 1946.068 & 1946.845 & 1948.145 \\
\hline
\end{tabular}


Shape of the observed histogram, estimated pdfs and cdfs of the compared distributions with data set 1 in (a) and (b) graphs below:

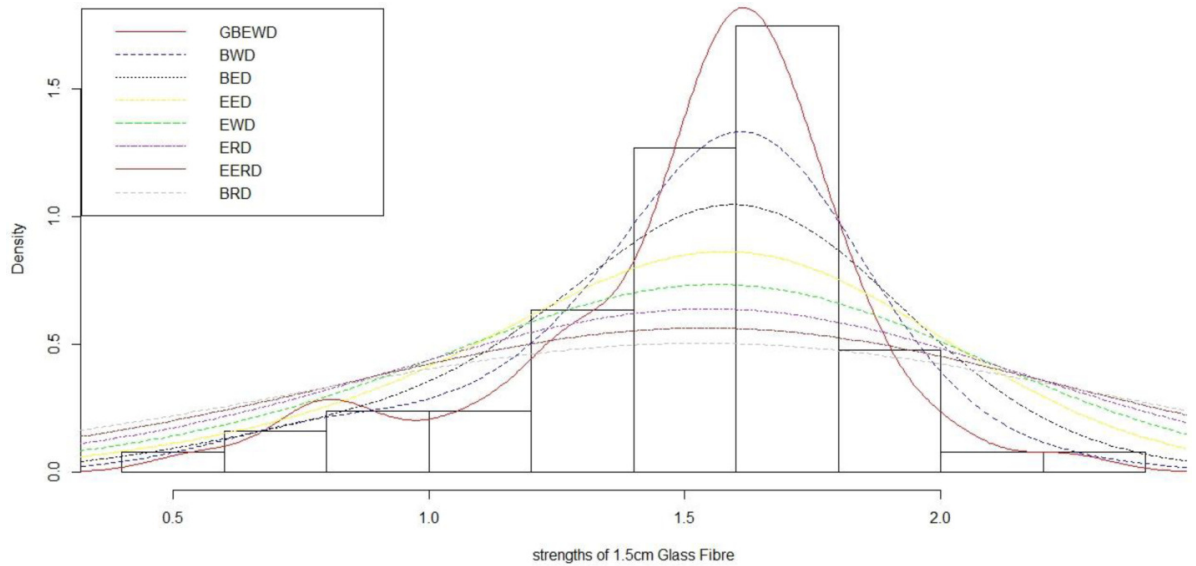

(a) Estimated PDFs

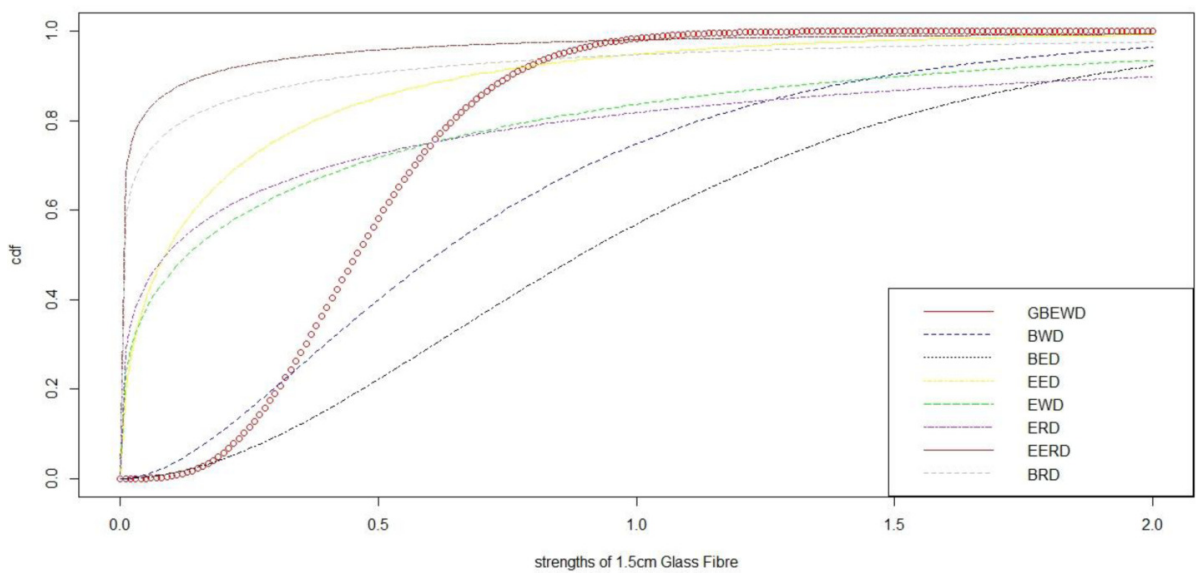

(b) Estimated CDFs

Figure 2. Consist graphs of the observed Histogram, estimated pdfs and cdfs of the GBEW distribution and compared distributions in different colours with data set 1 (the strengths of $1.5 \mathrm{~cm}$ glass fibres). 


\subsubsection{Data set 2}

The data set two for the second application was used by Handique and Chakraborty [14]. It consists of survival times (in years) for forty-six (46) group of patients given chemotherapy treatment. Also, in the second application, GBEW is compared with other generalized models listed in the first application.

Therefore, the values of MLEs and their corresponding standard errors (in parentheses) of the model parameters for the second data set are given in Table 4. Meanwhile, in Table 5, we compare the fits of the GBEW, BW, BE, EE, EW, ER, EER, and BR distributions. The values in Table 5 show that the generalized beta exponential Weibull (GBEW) model has the lowest values for the log-likelihood, AIC, CAIC, HQIC, and BIC statistics (for the first data set) among the fitted models. Hence, the GBEW model is the better fitted model among other competing models. While, Figure 3 depicts the plots of the observed histogram, estimated pdfs and cdfs for the distributions for data set 1 (survival times (in years) data). 
Table 4. MLEs of the parameters and their standard errors (in parentheses) for survival times (in years) data

\begin{tabular}{|c|c|c|c|c|c|c|}
\hline Model/Parameter & $\hat{a}$ & $\hat{b}$ & $\hat{c}$ & $\hat{\gamma}$ & $\hat{\hat{\lambda}}$ & $\hat{\theta}$ \\
\hline \multirow[t]{2}{*}{ GBEW $(\alpha, \beta, c, \gamma, \lambda, \theta)$} & 4.9750 & 0.0002 & 1.5560 & 2.4750 & 0.7493 & 0.6406 \\
\hline & (0.0118) & $(1.0022)$ & $(0.0000)$ & $(0.0000)$ & $(0.0000)$ & $(0.0000)$ \\
\hline \multirow[t]{2}{*}{$\mathrm{BW}(\alpha, \beta, 1, \gamma, 1, \theta)$} & 4.9730 & 3.8240 & 1 & 2.4650 & 1 & 0.6866 \\
\hline & $(0.0927)$ & $(0.0921)$ & & $(0.0010)$ & & $(0.0000)$ \\
\hline \multirow[t]{2}{*}{$\mathrm{BE}(\alpha, \beta, 1, \gamma, 1,1)$} & 4.9930 & 0.2340 & 1 & 2.4900 & 1 & 1 \\
\hline & $(0.0051)$ & $(0.3931)$ & & $(0.1641)$ & & \\
\hline \multirow[t]{2}{*}{$\mathrm{EE}(1,1, c, \gamma, \lambda, 1)$} & 1 & 1 & 1.3890 & 2.5570 & 0.5310 & 1 \\
\hline & & & $(0.1031)$ & $(0.0000)$ & $(0.0000)$ & \\
\hline \multirow[t]{2}{*}{$\mathrm{EW}(\alpha, 1, c, \gamma, 1, \theta)$} & 5.0490 & 1 & 1.0840 & 2.3770 & 1 & 0.7217 \\
\hline & $(0.0003)$ & & $(0.0030)$ & $(0.0002)$ & & $(0.0001)$ \\
\hline \multirow[t]{2}{*}{$\operatorname{ER}(1,1,1, \gamma, 1,2)$} & 1 & 1 & 1 & 2.5880 & 1 & 2 \\
\hline & & & & $(0.0000)$ & & \\
\hline \multirow[t]{2}{*}{$\operatorname{EER}(1,1, c, \gamma, \lambda, 2)$} & 1 & 1 & 1.5090 & 2.5883 & 0.5737 & 2 \\
\hline & & & $(0.0000)$ & (NA) & $(0.0000)$ & \\
\hline \multirow[t]{2}{*}{$\mathrm{BR}(\alpha, \beta, 1, \gamma, 1,2)$} & 4.9950 & 0.0070 & 1 & 2.5020 & 1 & 2 \\
\hline & (NA) & (NA) & & $(0.0001)$ & & \\
\hline
\end{tabular}

Table 5. Goodness-of-fit statistics for survival times (in years) data

\begin{tabular}{lccccc}
\hline Model & -2 Loglik & AIC & CAIC & HQIC & BIC \\
\hline GBEW & $\mathbf{1 8 3 3 . 8 2 6}$ & $\mathbf{3 6 7 9 . 6 5 2}$ & $\mathbf{3 6 8 1 . 8 0 6}$ & $\mathbf{3 6 8 3 . 7 6 2}$ & $\mathbf{3 6 9 0 . 6 2 4}$ \\
BW & 1858.800 & 3845.000 & 3727.036 & 3728.340 & 3732.915 \\
BE & 1891.100 & 3788.200 & 3789.277 & 3790.255 & 3793.686 \\
EE & 1901.220 & 3808.440 & 3809.011 & 3810.495 & 3813.926 \\
EW & 1911.320 & 3826.640 & 3826.919 & 3828.010 & 3830.297 \\
BR & 1914.540 & 3835.080 & 3835.651 & 3837.135 & 3840.566 \\
EER & 1919.500 & 3845.000 & 3845.571 & 3847.055 & 3850.486 \\
ER & 1941.010 & 3884.020 & 3884.111 & 3884.705 & 3885.849 \\
\hline
\end{tabular}


Shape of the estimated pdfs and cdfs of the compared distributions with data set 2 in (c) and (d) graphs below:

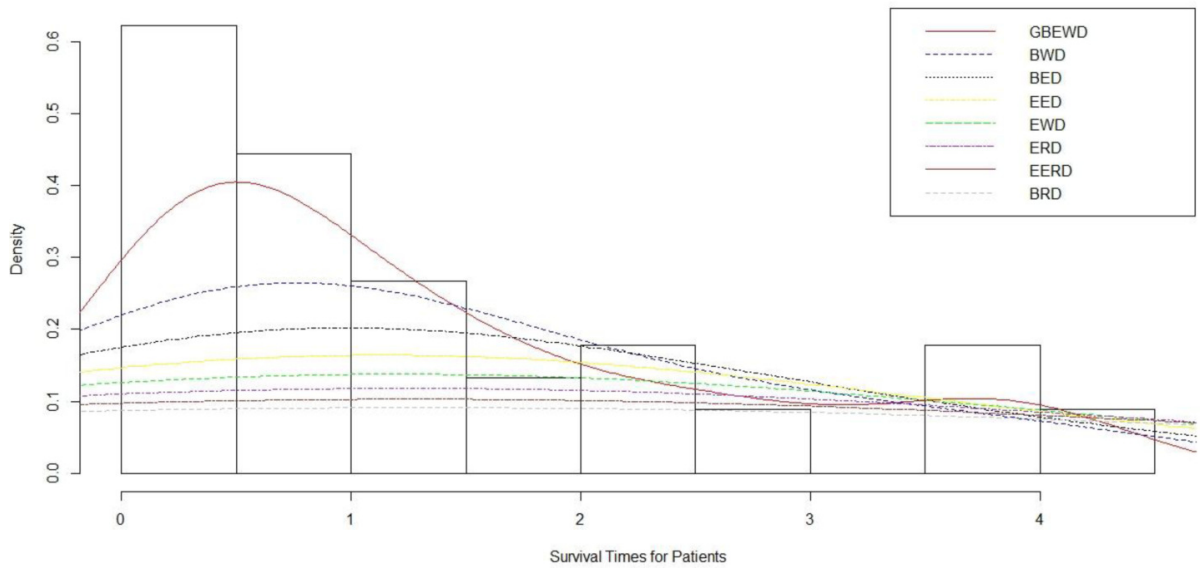

(c) Estimated PDFs

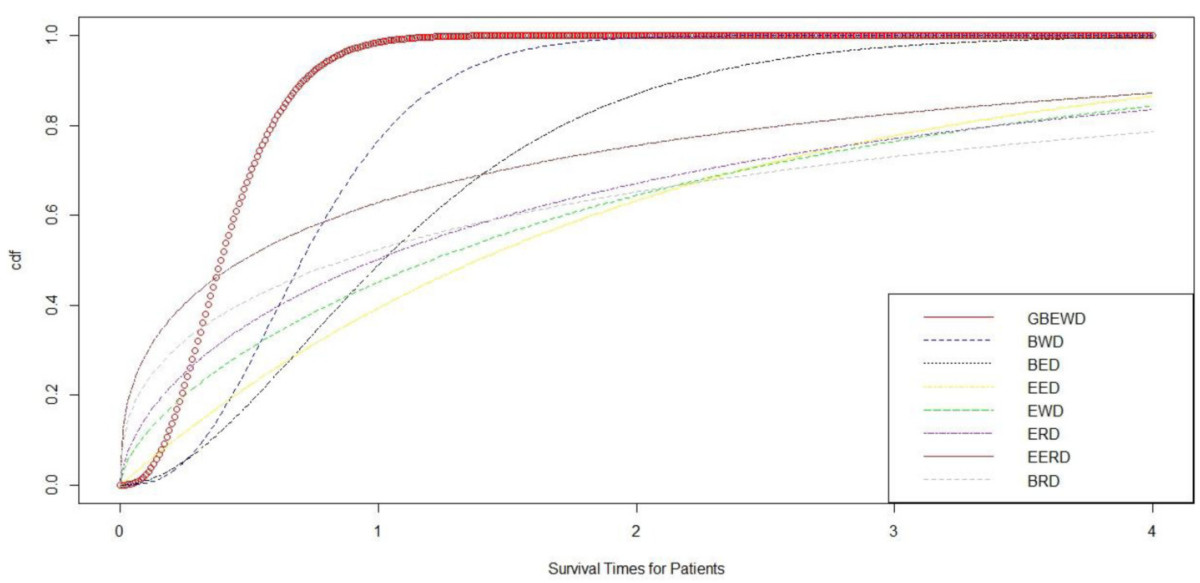

(d) Estimated CDFs

Figure 3. Contain plots of the observed Histogram, estimated pdfs and cdfs of the GBEW distribution and compared distributions in different colours with data set 2 (survival times (in years) for forty-six (46) group of patients given chemotherapy treatments). 


\section{Conclusion}

We investigate a distribution namely the generalized betaexponential Weibull distribution by adding two shape parameters, which generalizes the family of $T-X$ distribution called generalized beta exponential. Both density and hazard functions have different shapes and contains special case of distributions that have been proposed in literature such as beta-Weibull, beta exponential, exponentiated-Weibull and exponentiated-exponential distribution. Various properties of the distribution were obtained namely; moments, generating function, order statistics, Renyi entropy and Quantile function. Estimation of model parameters through maximum likelihood estimation method and observed information matrix are derived. Thereafter, the proposed distribution is illustrated with applications to two different real data sets. Finally, the GBEW distribution clearly shown that is better fitted to the two data sets than other competing distributions.

\section{References}

[1] M. Alizadeh, G. M. Cordeiro, E. de Brito and C. G. B. Demetrio, The beta MarshallOlkin family of distributions, Journal of Statistical Distributions and Applications 2(4) (2015), 1-18.

DOI: https://doi.org/10.1186/s40488-015-0027-7

[2] A. Alzaatreh, C. Lee and F. Famoye, A new method for generating families of continuous distributions, Metron 71(1) (2013), 63-79.

DOI: https://doi.org/10.1007/s40300-013-0007-y

[3] A. Alzaghal, F. Famoye and C. Lee, Exponentiated $T-X$ family of distributions with some applications, International Journal of Statistics and Probability 2(3) (2013), 31-49.

DOI: https://doi.org/10.5539/ijsp.v2n3p31

[4] S. K. Ashour and W. M. Afify, Estimation of the parameters of exponentiated Weibull family with type Ii progressive interval censoring with random removals, Journal of Applied Sciences Research 4(11) (2008), 1428-1442. 
[5] W. Barreto-Souza, A. H. S. Santos and G. M. Cordeiro, The beta generalized exponential distribution, Journal of Statistical Computation and Simulation 80(2) (2010), 159-172.

DOI: https://doi.org/10.1080/00949650802552402

[6] M. Bourguignon, R. B. Silva and G. M. Cordeiro, The Weibull-G family of probability distributions, Journal of Data Science 12(1) (2014), 5-68.

[7] A. Choudhury, A simple derivation of moments of the exponentiated Weibull distribution, Metrika 62(1) (2005), 17-22.

DOI: https://doi.org/10.1007/s001840400351

[8] G. M. Cordeiro and M. de Castro, A new family of generalized distributions, Journal of Statistical Computation and Simulation 81(7) (2011), 883-898.

DOI: https://doi.org/10.1080/00949650903530745

[9] G. M. Cordeiro, C. T. Cristino, E. M. Hashimoto and E. M. M. Ortega, The beta generalized Rayleigh distribution with applications to lifetime data, Statistical Papers 54(1) (2013), 133-161.

DOI: https://doi.org/10.1007/s00362-011-0415-0

[10] M. A. T. Elshahat, Approximate Bayes Estimators for the Exponentiated Weibull Parameters with Progressive Internal Censoring, The 20 ${ }^{\text {th }}$ Annual Conference on Statistics and Modeling in Human and Social Science, Faculty of Economic and Political Science, Cairo University, Egypt (2008), 123-136.

[11] F. Famoye, C. Lee and O. Olumolade, The beta Weibull distribution, Journal of Statistical Theory and Applications 4 (2005), 121-136.

[12] R. D. Gupta and D. Kundu, Exponentiated exponential family: An alternative to gamma and Weibull Distributions, Biometrical Journal 43(1) (2001), 117-130.

DOI: https://doi.org/10.1002/1521-4036(200102)43:1<117::AID-BIMJ117>3.0.CO;2-R

[13] H. M. Salem and M. A. Selim, The generalized Weibull-exponential distribution: Properties and applications, International Journal of Statistics and Applications 4(2) (2014), 102-112.

DOI: https://doi.org/10.5923/j.statistics.20140402.04

[14] Laba Handique and Subrata Chakraborty, Beta generated Kumaraswamy- $G$ and other new families of distribution, arXiv preprint arXiv:1603.00634 (2016), 1-34.

[15] J. F. Kenney and E. S. Keeping, Mathematics of Statistics, 101-102, Part 1, $3^{\text {rd }}$ Edition, Princeton, NJ, 1962.

[16] C. Kim, J. Jung and Y. Chung, Bayesian estimation for the exponentiated Weibull model type II progressive censoring, Statistical Papers 52(1) (2011), 53-70.

DOI: https://doi.org/10.1007/s00362-009-0203-2 
[17] C. Lee, F. Famoye and O. Olumolade, Beta-Weibull distribution: Some properties and applications to censored data, Journal of Modern Applied Statistical Methods 6(1) (2007), 173-186.

DOI: https://doi.org/10.22237/jmasm/1177992960

[18] M. A. W. Mahmoud and M. G. M. Ghazal, Estimations from the exponentiated Rayleigh distribution based on generalized type-II hybrid censored data, Journal of Egyptian Mathematical Society 25(1) (2017), 71-78.

DOI: https://doi.org/10.1016/j.joems.2016.06.008

[19] E. Mahmoudi, The beta generalized Pareto distribution with application to lifetime data, Mathematics and Computers in Simulation 81(11) (2011), 2414-2430.

DOI: https://doi.org/10.1016/j.matcom.2011.03.006

[20] A. L. Morais, G. M. Cordeiro and Audrey H. M. A. Cysneiros, The beta generalized logistic distribution, Brazilian Journal of Probability and Statistics 27(2) (2013), 185-200.

\section{DOI: https://doi.org/10.1214/11-BJPS166}

[21] J. J. A. Moors, A quantile alternative for kurtosis, Journal of the Royal Statistical Society: Series D 37(1) (1988), 25-32.

DOI: http://dx.doi.org/10.2307/2348376

[22] S. Nadarajah and S. Kotz, The beta exponential distribution, Reliability Engineering and System Safety 91(6) (2006), 689-697.

DOI: https://doi.org/10.1016/j.ress.2005.05.008

[23] M. M. Nassar and F. H. Eissa, On the exponentiated Weibull distribution, Communications in Statistics-Theory and Methods 32(7) (2003), 1317-1336.

DOI: https://doi.org/10.1081/STA-120021561

[24] M. M. Nassar and F. H. Eissa, Bayesian estimation for the exponentiated Weibull model, Communications in Statistics-Theory and Methods 33(10) (2005), 2343-2362.

DOI: https://doi.org/10.1081/STA-200031447

[25] P. E. Oguntunde, O. S. Balogun, H. I. Okagbue and S. A. Bishop, The Weibullexponential distribution: Its properties and applications, Journal of Applied Sciences 15(11) (2015), 1305-1311.

DOI: https://doi.org/10.3923/jas.2015.1305.1311

[26] O. C. Okoli, G. A. Osuji, D. F. Nwosu and K. N. C. Njoku, On the modified extended generalized exponential distribution, European Journal of Statistics and Probability 4(4) (2016), 1-11.

[27] A. K. Olapade, On extended generalized exponential distribution, British Journal of Mathematics and Computer Science 4(9) (2014), 1280-1289.

DOI: https://doi.org/10.9734/BJMCS/2014/8191 
[28] M. Pal, M. M. Ali and J. Woo, Exponentiated Weibull distribution, Statistica 66(2) (2006), 139-147.

DOI: https://doi.org/10.6092/issn.1973-2201/493

[29] R. R. Pescim, C. G. B. Demétrio, G. M. Cordeiro, E. M. M. Ortega and M. R. Urbano, The beta generalized half-normal distribution, Computation Statistics and Data Analysis 54(4) (2010), 945-957.

DOI: https://doi.org/10.1016/j.csda.2009.10.007

[30] A. M. Salem and O. E. Abo-Kasem, Estimation for the parameters of the exponentiated Weibull distribution based on progressive hybrid censored samples, International Journal of Contemporary Mathematical Sciences 6(35) (2011), 1713-1724.

[31] R. L. Smith and J. C. Naylor, A comparison of maximum likelihood and Bayesian estimators for the three-parameter Weibull distribution, Journal of the Royal Statistical Society: Series C 36(3) (1987), 358-369.

DOI: https://doi.org/10.2307/2347795

[32] N. Singla, K. Jain and S. K. Sharma, The beta generalized Weibull distribution: Properties and applications, Reliability Engineering System Safety 102 (2012), 5-15.

DOI: https://doi.org/10.1016/j.ress.2012.02.003

[33] L. Zea, R. Silva, M. Bourguignon, A. Santos and M. Corderio, The beta exponentiated Pareto distribution with application to bladder cancer susceptibility, International Journal of Statistics and Probability 1(2) (2012), 8-19.

DOI: https://doi.org/10.5539/ijsp.v1n2p8 